\title{
Anatomical and Functional Preliminary Results of Total Non-Cemented Hip Prostheses
}

\author{
A. M. Abdoul Wahab ${ }^{1 *}$, M. Koini², B. Dembele, A. D. Sane³, A. N'Diaye ${ }^{3}$, C. Dieme ${ }^{3}$, N. F. Coulibaly ${ }^{3}$ \\ ${ }^{1}$ National Hospital of Niamey, Niamey, Niger \\ ${ }^{2}$ Regional Hospital of Maradi, Maradi, Niger \\ ${ }^{3} \mathrm{CHU}$ Aristide LE-DANTEC of Dakar, Dakar, Senegal \\ Email: ${ }^{*}$ medwahabe@gmail.com
}

How to cite this paper: Abdoul Wahab, A.M., Koini, M., Dembele, B., Sane, A.D., N'Diaye, A., Dieme, C. and Coulibaly, N.F. (2017) Anatomical and Functional Preliminary Results of Total Non-Cemented Hip Prostheses. Open Journal of Orthopedics, 7, 329-336.

https://doi.org/10.4236/ojo.2017.710033

Received: September 11, 2017

Accepted: October 21, 2017

Published: October 24, 2017

Copyright (C) 2017 by authors and Scientific Research Publishing Inc. This work is licensed under the Creative Commons Attribution-NonCommercial International License (CC BY-NC 4.0). http://creativecommons.org/licenses/by-nc/4.0/

\begin{abstract}
The aim of this work was to assess the preliminary anatomical and functional results of patients to establish prospects. Materials and method: We report here the preliminary results of a retrospective mono-centric, homogeneous and continuous series, of 21 non-cemented total hip implants, implanted from January 2011 to December 2014. The mean follow-up time was 24 months. The patients were evaluated by Harris's hip at the last follow-up. Results: The mean age of the intervention was 30 years. In our series, we observed a male predominance ( $60 \%$ of cases). Clinically, the Harris score varied significantly from $52.6 \pm 11.4$ pre-operatively to $85.1 \pm 5.1$ during the last follow-up visit. Among the items in the score, pain was the parameter that showed the greatest improvement. Thigh pain was observed in only one patient operated on the two sides. At the level of radiology, the femoral implant showed great stability. Bone resorption was suspected in 14 cases (66.66\%), and confirmed in 4 cases (19.04\%) with excellent bone remodeling, illustrated by the Engh and Massin scores. A loosening and a prosthetic dislocation was observed in a patient (4.76\%). Any heterotopic ossification was observed. Conclusion: Our study confirmed good clinical and radiological results of the non-cemented hip prosthesis series in young patients. The rates of aseptic loosening, acetabular edges or osteolysis are low and stable over time.
\end{abstract}

\section{Keywords}

Hip, Total Non-Cemented Prostheses

\section{Introduction}

Total non-cemented hip arthroplasty with metal-polyethylene torques remains the benchmark despite osteolysis induced by polyethylene wear, which remains 
the main factor of failure in the long term. Young and active patients undergoing a total hip arthroplasty are most at risk of aseptic loosening. Since the origin of the total hip arthroplasty, the discussion of the method of fixing the implants, with or without cement, remains as everlasting.

Around the 1980s, the addition of a coating of hydroxyapatite represents a "third way" that claims its specificity [1].

Non-cemented prostheses seek biological binding by bone regrowth around the implant. The choice of a non-cemented arthroplasty is based both on its ability to allow a perfect primary fixation to facilitate its osseointegration and its ability to avoid the onset and progression of secondary osteolysis. The hydroxyapatite has shown its efficacy in terms of histologic or radiological osseointegration [1] [2].

The objective of this work is:

- To assess the preliminary anatomical and functional results of the patients to establish prospects.

\section{Materials and Method}

\subsection{Type and Period of the Study}

This is a retrospective clinical study carried out at the Aristide LE DANTEC University Hospital Center, covering 21 patients all of which are total cementless hip prostheses. It is a bi-operator series carried out between January 2011 and December 2014. The mean follow-up is 24 -month post-operation.

\subsection{Selection Criteria}

The inclusion criteria were: any patient who benefited from placing a stem and a cementless acetabulum, whatever the indication of the orthopedic and traumatology services of the Aristide LE DANTEC hospital.

The patients excluded from the study were:

- All cemented hip prostheses;

- Hybrid prostheses;

- The patients, whose prosthesis was redone;

- Patients lost to follow-up;

- The deceased patient;

- And incomplete medical records.

\subsection{Source of Data}

Patients were re-examined after 6 months, 1 year and 2 years, and then contacted by telephone for a functional assessment of their hips. Functional hip score according to Postel-Merle d'Aubigné.

The Harris score gave us a second score to evaluate our patients during the last follow-up.

The radiological evaluation was based on the radiology images of the narrow frontal pelvis as well as radiographs of the operated hip on the face taking the 
entire prosthesis.

For the sake of simplicity, the radiological study was carried out using three $\mathrm{X}$-rays from the frontal pelvis: pre-operation, post-operation and at the last follow-up.

The radiological criteria were:

- The positioning of the implant

- The inclination of the implant on the horizontal was measured with respect to the bi-ischial line, which made it possible to obtain for each implant an immediate post-operation alpha angle and an angle alpha at the recoil. Any variation in the alpha $>3^{\circ}$ angle would result in migration of the implant. The angle of inclination of the cup relative to the bi-ischial line is normally $45^{\circ}$.

- The acetabular edges were studied in the three zones defined by De Lee and Charnley to make the diagnosis of loosening, these edges were studied according to their localisation, their size and their evolutionary character.

- The elongation was measured by the vertical distance between the radiographic $U$ and the top of the small trochanter before and after the surgery.

- Peri-prosthetic ossification was evaluated according to the Brooker classification.

\subsection{Data Analysis}

The analysis and the data entry were made using soft software Word 13, Excel 13, Epi info version 7.0.

\subsection{Bias and Limits}

This was a retrospective study on patients' files and reviews, and it is therefore subject to certain methodological criticisms.

There is thus a recruitment bias due to the multiplicity of aetiologies, or the high number of patients contacted by telephone who do not fully understand their pain and estimate their daily activities.

This work corresponds to a bi-operator series but mono-centric, which can influence the quality of the result. This represents a performance bias.

The susceptibility bias is well controlled by the implantation of a single implant in all the patients of the series (cementless prosthesis).

The size of the sample studied is greater than 20 , allowing the adoption of the hypothesis of a normal distribution and therefore using the conventional statistical tests.

The data collector was different from the operators and was neither a developer nor a designer of implanted equipment. This should in principle increase the objectivity of the study.

We decided to compare this work to different recent series of cementless hip arthroplasty using different techniques or not, but in which there was the homogeneity of the implants. 


\subsection{Follow-Ups}

The mean follow-up of this series was 24 months.

\section{Results}

The mean age at intervention was 30 years; the age group 21 up to 27 was the most affected (Table 1). In our series we note a male predominance with $60 \%$ of cases (Table 2). Clinically, in our series, the mean total PMA score at the last follow-up was 17.2 (Table 3 ) and the Harris score varied significantly from 52.6 \pm 11.4 pre-operatively to $85.1 \pm 5.1$ at the last recoil visit (Table 4). Among the items in the score, pain was the parameter that showed the greatest improvement. Thigh pain, often associated with cementless stems, was observed in only one patient operated on two sides. At the radiological level, the femoral implant showed great stability (Figure 1). The radiographic analysis was found in almost all cases (85\%, moderate bone resorption of calcar without true osteolysis).

- No borderline was found in the immediate postoperative period, or at the last follow-up within the implants.

- We didn't find osteolysis, either expansive or extensive, or multifocal. The wear of polyethylene could not be evaluated due to the lack of means to this end. We did not observe heterotopic ossification.

- Thus, radiological refocusing of the head showed that

- The prosthetic cup was medialized by $2.57 \mathrm{~mm}$;

- It was placed high of $4.09 \mathrm{~mm}$;

- The femoral offset was decreased by $1.94 \mathrm{~mm}$;

- A $1.49 \mathrm{~mm}$ elongation at the lower limb with the prosthesis was noted.

Table 5 shows the average values of the recentering of the head (Table 5).

We used the fixation and stability score of the femoral stem according to Engh and Massin.

Table 1. Distribution of patients by age group.

\begin{tabular}{ccc}
\hline Age range & Number & Percentage \\
\hline 18 to 21 & 3 & $15 \%$ \\
21 to 27 & 8 & $45 \%$ \\
28 to 34 & 2 & $10 \%$ \\
35 to 41 & 3 & $15 \%$ \\
42 to 48 & 2 & $10 \%$ \\
49 to 55 & 1 & $5 \%$ \\
\hline
\end{tabular}

Table 2. Distribution by gender.

\begin{tabular}{ccc}
\hline Gender & Number & Percentage \\
\hline Men & 12 & $60 \%$ \\
Women & 8 & $40 \%$ \\
\hline
\end{tabular}


Table 3. Pre-operative and postoperative PMA distribution.

\begin{tabular}{lcc}
\hline & & PMA \\
\cline { 2 - 3 } PRE-OPERATORY & Minimum & 3 \\
& Average & 7.9 \\
& Maximum & 13 \\
& Minimum & 11 \\
POST-OPERATORY & Average & 17.2 \\
& Maximum & 18 \\
\hline
\end{tabular}

Table 4. pre-operative Harris score and at last follow-up.

\begin{tabular}{cccc}
\hline Median scores & Pre-operative & At last follow-up & $\mathbf{P}$ \\
\hline Harris Global & $\mathbf{5 2 . 6} \pm \mathbf{1 1 . 4}$ & $\mathbf{8 5 . 1} \pm \mathbf{5 . 1}$ & $<0.001$ \\
Harris Pain & $19.2 \pm 1.2$ & $39.4 \pm 3.2$ & $<0.001$ \\
Mobility Harris & $2.5 \pm 0.8$ & $3.5 \pm 0.6$ & $<0.001$ \\
Walk Harris & $19.4 \pm 1.2$ & $22.3 \pm 0.4$ & $<0.001$ \\
Activity Harris & $6.3 \pm 1.2$ & $8.1 \pm 0.6$ & $<0.001$ \\
\hline
\end{tabular}

Table 5. the average value of the re-centering of the head.

\begin{tabular}{cccc}
\hline Type & $\begin{array}{c}\text { Prosthesis side at last } \\
\text { follow-up }\end{array}$ & Non-prosthesis side & Difference \\
\hline $\mathrm{X}$ & $34.48 \mathrm{~mm}$ & $37.05 \mathrm{~mm}$ & $-2.57 \mathrm{~mm}$ \\
$\mathrm{Y}$ & $22.34 \mathrm{~mm}$ & $18.25 \mathrm{~mm}$ & $4.09 \mathrm{~mm}$ \\
$\mathrm{Z}$ & $46.10 \mathrm{~mm}$ & $48.04 \mathrm{~mm}$ & $-1.94 \mathrm{~mm}$ \\
$\mathrm{~W}$ & $60.55 \mathrm{~mm}$ & $59.06 \mathrm{~mm}$ & $1.49 \mathrm{~mm}$ \\
\hline
\end{tabular}

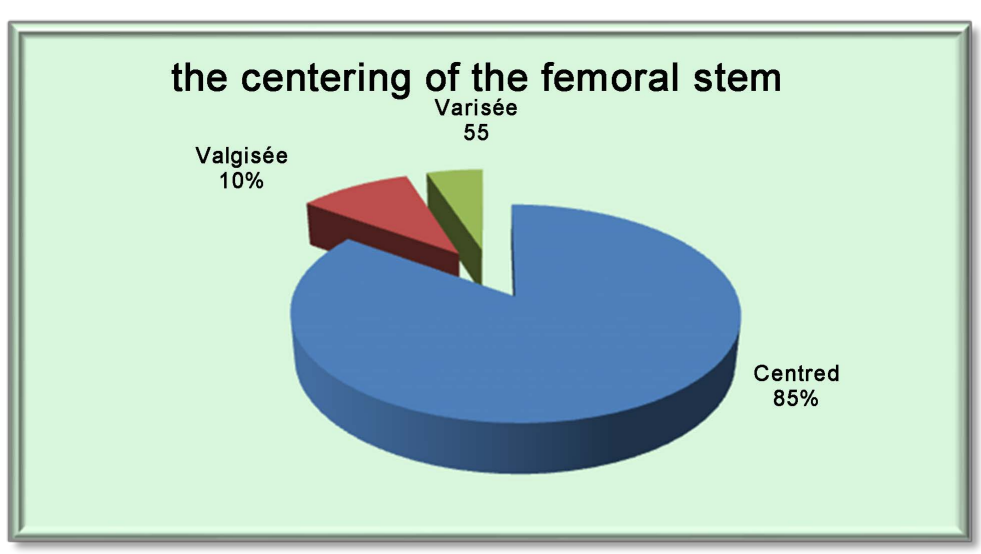

Figure 1. Distribution according to the centering of the femoral stem.

The analysis according to the score of Engh and de Massin was carried out from the analysis of pelvis radiographs from the face to the last recoil, for 21 
prostheses. A stem was considered unstable. Bone rehabilitation is suspected in 14 cases $(66.66 \%)$, and confirmed in 4 cases (19.04\%). Illustrated by Engh and Massin score (Table 6).

Table 6. Fixation score according to Engh and Massin.

\begin{tabular}{ccc}
\hline Fixing Score & Number & Percentage \\
\hline $\begin{array}{c}\text { Bone reaffirmation confirmed }(\geq 10 \mathrm{pts}) \\
\text { Suspected bone retention }(0<\mathrm{n}<10 \mathrm{pts})\end{array}$ & 4 & 19.04 \\
Fibrous encapsulation $(-10<\mathrm{n}<0)$ & 0 & 0 \\
Unstable $(<-10)$ & 1 & 4.76 \\
\hline
\end{tabular}

\section{Discussion}

The average age of intervention in our series was 30 years. In the different series we have cited, the average age was more often 50 years or more [3].

We can explain this early age in our series by the fact that in $35 \%$ of cases, it was a sickle-cell osteonecrosis of the femoral head thus dominating the cases of identified aetiologies.

In our series we noted a male predominance $(60 \%)$. Our results were in line with those of literature, with $58.2 \%$ of men in the Bonnomet F series [2], 78.8\% of men in D. Pourreyron's series [4], and 55.2\% of men in the series of A. Fiquet [5].

Clinical results were assessed on Merle d'Aubigné and Postel, and Harris scores. These two scores are based on the clinical evaluation of three parameters: pain, mobility and functional impairment. In our series, the mean total PMA scores and the mean total HHS scores at the last follow-up were respectively 17.2 and 85.1. These are superimposed on the data of literature. If we compare the results of our series to the series of prostheses having only a hydroxyapatite coating (but a different friction torque) [3] [4] [6], their average PMA at the last follow-up was 16.95 and their Harris Score was 94. Again, overall, the results were similar.

Radiological findings were assessed according to the Engh-Massin Score. We found no reactive line around the hydroxyapatite-coated part of the femoral stem, which could be interpreted as a favorable sign of integration of the femoral stem. $15 \%$ of the femoral stems have a border of less than $2 \mathrm{~mm}$ limited to zones 7 and 1 . In the case of acetabulum, it was found in 2 cases (10\%), a border less than $2 \mathrm{~mm}$, non-evolutionary between the immediate post-operation radiograph and the last follow-up. None of these patients complained about hip pain. A single acetabulum shows evolution, with the appearance of an acetabular loosening and a prosthetic dislocation. Indeed, the patient presents a bad clinical result, with the appearance of pain in his hip. A short-term cotyloid change should be considered. Finally, it should be noted that in $15 \%$ of cases, a border visible at the acetabular level in the immediate post-operation disappeared on X-rays at 
the last follow-up. This is probably due to imperfect milling and the osteoinductive effect of hydroxyapatite.

Haut duThe analysis of the cortical changes around the femoral stem showed that in our series there is a cortical hypertrophy with a type of stress shielding at the end of the stem only $5 \%$ of the cases, which is less than what one finds in the literature. In our series, none of the femoral stems shows signs of osteolysis or cortical cavity. No osteolysis was found, neither expansive nor multifocal at the level of the femoral stem throughout all the series [7].

The study of radiographic scores showed a constant bone regrowth on all the femoral stems of the series, indeed a single femoral prosthesis was considered unstable according to the Engh-Massin score. The favorable results of the use of hydroxyapatite-coated stems are reported in the literature [8] [9].

\section{Conclusion}

Our study confirms the good clinical and radiological results of the non-cemented hip prosthesis series in young patients. The rates of aseptic loosening, acetabular edges or osteolysis are low and stable over time.

\section{Conflicts of Interests}

The authors claim no conflicts of interest regarding this paper.

\section{Authors Who Contributed}

A. M. Abdoul Wahab*, M. Koini, A. D. Sane, A N'diaye, C. Dieme, N. F. Coulibaly.

\section{References}

[1] Brooker, A.F., Bowerman, J.W., Robinson, R.A. and Rileh Jr., L.H. (1973) Ecto-Pik Ossification Following Total Hip Replacement. Incidence and a Method of Classification. The Journal of Bone and Joint Surgery, American Volume, 55-A, 1629-1632. https://doi.org/10.2106/00004623-197355080-00006

[2] Bonnomet, F., Ehlinger, M., Molina, V. and Thomazeau, H. (2006) Periprosthetic Fractures around the Hip and Knee. Classification of Fractures on Hip Prosthesis. Revue de Chirurgie Orthopedique, 92, 2S51-2S56.

[3] Plotard, F., et al. (2008) Overall Results of a Series of 941 First-Line Implanted Arthroplasties. Orthopedic and Repair Surgery of the Motor Apparatus, 94S, S142-S145.

[4] Pourreyron, D., Ayadi, H. and Bonnomet, F. (2008) Total Hip Prosthesis in Patients under 30 Years of Age: Presentation of the Series and Method of Analysis. Review of Orthopedic and Repair Surgery of the Motor Apparatus, 94S, S135-S137.

[5] Fiquet, A. and Walnut, D. (2008) Total Hip Prosthesis with Double Mobility and Surgery and Minimally Invasive Maîtrise Orthopédique. No. 173, April.

[6] Cohen, G. and Rosset, P. (2009) Results of 76 Total Non-Cemented Hip Implants with Hydroxyapatite Complete Coating with a Minimum Follow-Up of Five Years. Review of Orthopedic and Traumatologic Surgery, 95S, S74-S84.

[7] Beldame, J., et al. (2009) Osteolysis and Total Metal-to-Metal Hip Arthroplasty with 
Unsealed Cup. About 106 Cases. Review of Orthopedic and Traumatological Surgery, 95, 579-592.

[8] Druon, J., Aubault, M., Le Nail, L. and Rosset, P. (2013) Retractable Femoral Stem with Hydroxylapatite Cover, Modular and with Distal Locking. Prospective Series of 47 Stems with a Minimum Setback of Five Years. Review of Orthopedic and Traumatologic Surgery, 99, S72-S76.

[9] Portet, Y. and Reina, N. (2016) Comparative Study of Cemented vs. Cementless Hip Implants in Elderly Patients with Femoral Neck Fracture. Revue de Chirurgie Orthopédique et Traumatologique, 102, S73-S190. 